\section{Le facteur de survie neuronale GDNF}

\section{Un nouveau modulateur \\ de la signalisation sémaphorine \\ au cours de la navigation axonale}

Camille Charoy, Valérie Castellani
Centre de génétique et de physiologie moléculaire et cellulaire (CGphiMC), UMR CNRS 5534 université de Lyon, UCBLI, 16, rue Raphaël Dubois, 69622 Villeurbanne, France. valerie.castellani@univ-lyonl.fr

\section{Le GDNF : facteur de croissance et chimioattractant pour les axones}

C'est dans le cadre d'études visant à purifier des molécules sécrétées par les cellules gliales et capables de promouvoir la survie neuronale que le facteur neurotrophe GDNF (glial cell line-derived neurotrophic factor) a été isolé par $\mathrm{N}$. Bermingham et ses collaborateurs [1]. La structure de cette petite protéine glycosylée, d'une quarantaine de kDa, l'apparente aux molécules de la superfamille du TGF $\beta$ (transforming growth factor $\beta$ ) et forme, avec trois autres membres - la neurturine [NRN], l'artémine [ART] et la persephine [PSP] - la famille des GDNF. Le GDNF est exprimé dans de nombreux tissus de l'organisme comme le foie, le cœur, les poumons ou encore les testicules. Dans les systèmes nerveux central et périphérique, il est détecté dans la plupart des structures cérébrales. II est ainsi accessible à de nombreuses populations de neurones: neurones dopaminergiques du mésencéphale ou cellules de Purkinje, motoneurones de la moelle épinière et, en périphérie, neurones sympathiques et neurones entériques dérivés des crêtes neurales [2]. Le GDNF possède des propriétés biologiques diversifiées: dans le cerveau adulte, un de ses effets majeurs est de promouvoir la survie des neurones dopaminergiques. Il régule également d'autres aspects importants de la physiologie des neurones dopaminergiques, par exemple en stimulant la synthèse et le turn-over de la dopamine. Dans la moelle épinière et les ganglions périphériques, il exerce des effets neuroprotecteurs et neurotrophiques similaires sur les motoneurones et les neurones sensoriels. Le GDNF est ainsi une cible thérapeutique de première importance dans le contexte des maladies neurodégénératives. II est notamment l'objet d'une attention particulière dans la recherche de traitements de la maladie de Parkinson [3].

Au cours du développement, le GDNF participe à de nombreuses étapes de la formation du système nerveux. II régule la prolifération, la différenciation et la survie neuronales. Il participe aussi au contrôle des migrations cellulaires et à la navigation des projections neuronales. On attribuait jusqu'à maintenant au GDNF un rôle de facteur promoteur de croissance axonale et de chimioattractant dans ce processus de formation des connexions nerveuses, fonctions mises en évidence lors d'études de navigation des projections périphériques $[2,4]$. C'est en étudiant les mécanismes de navigation d'une population d'axones se projetant dans la moelle épinière, les projections commissurales, que nous avons découvert un nouveau rôle du GDNF.

La navigation des projections axonales commissurales de la moelle épinière

La plaque du plancher, un territoire de cellules gliales situé au bord ventral du canal central de la moelle épinière, est un centre organisateur majeur, à la fois pour la mise en place des sous-types neuronaux de la moelle épinière et pour la formation des connexions spinales. La plaque du plancher sécrète des morphogènes qui contribuent à définir les identités des neurones spinaux et qui sont réutilisés plus tardivement pour orienter la trajectoire des projections spinales. C'est par exemple le cas de Shh (sonic hedgehog) et des Wnt (wingless integration site), qui jouent des rôles clefs dans le guidage des projections commissurales [5]. Ces projections émanent d'une population d'interneurones dorsaux et sont guidées jusque dans la plaque du plancher. À ce niveau, elles traversent la ligne médiane pour se projeter ensuite sur des neurones cibles situés du côté opposé à la région de leurs corps cellulaires d'origine. Ces projections font partie d'un ensemble de circuits, dits commissuraux ou controlatéraux, qui permettent l'interconnexion des parties droite et gauche du système nerveux central assurant ainsi une coordination et une intégration des informations et des commandes [6]. La plaque du plancher fonctionne également comme une barrière, en repoussant les populations de projections avoisinantes, dites ipsilatérales, qui sont destinées à innerver des neurones situés du même côté.

Le système des projections commissurales est l'un des modèles d'étude les plus exploités par les scientifiques pour comprendre les mécanismes qui contrôlent la navigation axonale, et identifier les molécules de guidage qui sont lues par l'extrémité motile de l'axone (qu'on appelle le cône de croissance) et l'orientent en direction de sa cible. De très nombreux travaux ont révélé qu'au cours de la navigation des projections commissurales, les cônes de croissance répondent à une combinaison de signaux de guidage qui contrôlent 


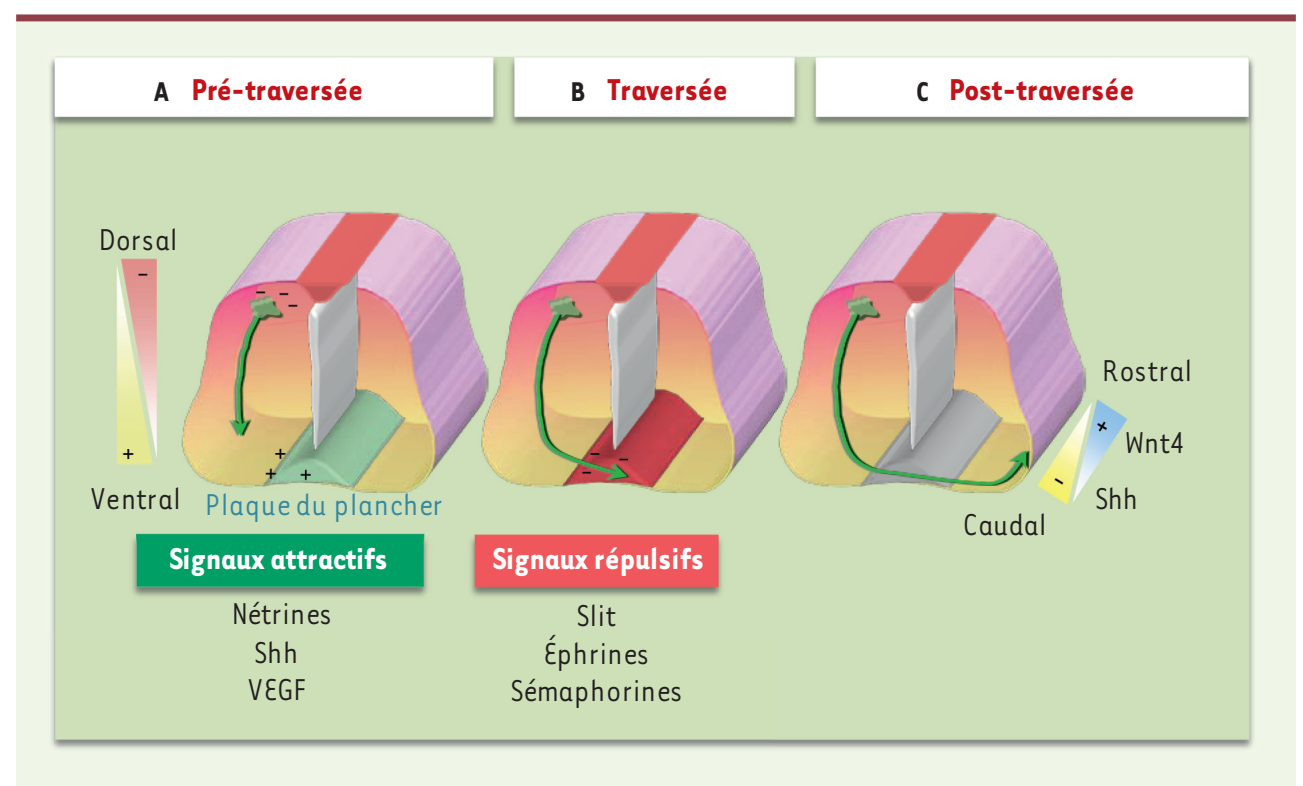

Figure 1. Représentation schématique des trois étapes clefs de la navigation des projections commissurales de la moelle épinière au travers de la ligne médiane. A. Étape de pré-traversée. Les axones commissuraux des interneurones dorsaux s'orientent en direction ventrale grâce à l'expression de signaux répulsifs dorsaux, et de signaux attractifs sécrétés par la plaque du plancher : les nétrines, Shh et le VEGF. B. Étape de traversée. Une fois la ligne médiane franchie, les axones acquièrent une réponse à des signaux répulsifs de la plaque : les Slit, les éphrines et les sémaphorines. Ce mécanisme transforme la plaque, initialement permissive, en un territoire hostile, qui repousse les axones du côté opposé de la moelle. C. Étape de post-traversée. La traversée de la plaque achevée, les axones commissuraux tournent en direction rostrale grâce à des gradients rostro-caudaux de morphogènes ou molécules de guidage, les Wnt et Shh, et progressent en direction de leur territoire cible.

successivement les étapes clés de leur trajet: l'étape de pré-traversée, qui amène les axones commissuraux dans la plaque du plancher; l'étape de traversée, au cours de laquelle les axones traversent la ligne médiane; et l'étape de post-traversée, qui permet aux axones de sortir de la plaque du plancher et de tourner longitudinalement pour naviguer parallèlement à la ligne médiane en direction du cerveau (Figure 1).

\section{Régulation des réponses axonales aux signaux de guidage}

Un des paramètres importants de cette navigation réside dans la régulation temporelle de la perception des signaux de guidage présents dans la plaque du plancher. En effet, pour que la traversée de la ligne médiane s'accomplisse, il est nécessaire que, dans un premier temps, les axones commissuraux perçoivent des signaux (signaux attractifs) qui les attirent dans la plaque du plancher, de biochimie, notre équipe a caractérisé en 2010 le mécanisme contrôlant le gain de réponse à un signal répulsif de la plaque du plancher, la sémaphorine 3B (Sema3B). Ce mécanisme module la disponibilité du récepteur de Sema3B à la surface du cône de croissance. Ce récepteur est un complexe formé de deux sous-unités : la neuropiline-2 (Nrp2) qui fixe le ligand, et la plexine-Al (PlexAl) qui est recrutée par la neuropiline-2 pour assurer la transduction du signal Sema3B répulsif dans le cône de croissance. Au stade de pré-traversée, les deux sous-unités du récepteur sont synthétisées par les neurones commissuraux, mais seule Nrp2 est présente à la surface du cône de croissance. En effet, PlexAl est clivée par puis, et seulement dans un deuxième temps, des signaux répulsifs qui les repoussent hors de la plaque du plancher et les dirigent dans l'hémi-moelle opposée. Les principaux acteurs moléculaires de ces effets d'attraction et de répulsion exercés par la plaque du plancher ont été caractérisés. Les nétrines, Shh et le VEGF (vascular endothelial growth factor) agissent ensemble pour attirer les axones commissuraux dans la plaque, tandis que les Slit, les éphrines et les sémaphorines entrent en action dès leur traversée de la ligne médiane pour repousser les axones hors de la plaque du plancher [6, 7] (Figure 1). En revanche, les mécanismes moléculaires qui contrôlent les variations séquentielles de sensibilité des neurones à ces deux types de signaux sont encore très peu connus. Par des approches expérimentales combinant différents modèles murins et aviaires, des modèles de cultures de tissus et des techniques une protéase endogène, la calpaïne 1 , ce qui bloque la formation d'un récepteur fonctionnel et donc la lecture du signal Sema3B par le cône de croissance. Au stade de traversée, le cône de croissance est exposé à des signaux émanant de la plaque du plancher qui suppriment l'activité calpaïne, et donc le clivage de PlexAl. La sous-unité parvient à la surface du cône de croissance, où elle s'associe à Nrp2, confèrant une sensibilité au signal répulsif Sema3B. Nous avons identifié NrCAM (neuronal cell adhesion molecule), une molécule d'adhésion de la superfamille des immunoglobulines, comme étant un premier signal de la plaque capable d'inhiber l'activité calpaïne et de sensibiliser le cône de croissance à Sema3B. Cependant, l'absence de cette molécule chez l'embryon de souris n'entraînait aucunement le spectre des défauts des projections commissurales que l'on pouvait attendre compte tenu de l'altération du mécanisme de gain de 


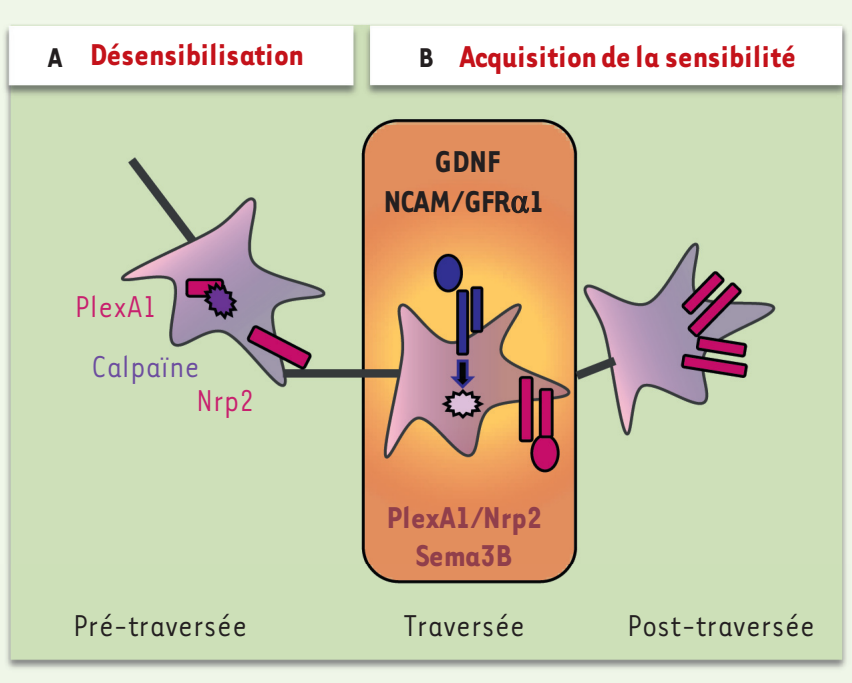

Figure 2. Action du GDNF dans le mécanisme de gain de réponse au signal répulsif Sema3B. A. Mécanisme de désensibilisation. Pendant l'étape de pré-traversée, le corécepteur PlexAl est clivé par la calpaïne 1 , ce qui empêche l'assemblage d'un récepteur fonctionnel de Sema3B sur le cône de croissance. Ce dernier est donc insensible à l'effet répulsif de Sema3B, et entre dans la plaque du plancher. B. Mécanisme de sensibilisation. Pendant la traversée, le GDNF se lie à son récepteur NrCAM/GFR $\alpha$ l porté par le cône de croissance, et active une signalisation qui inhibe l'activité de la calpaïne 1 . PlexAl est alors accessible à la surface du cône de croissance, et le récepteur de Sema3B, devenu fonctionnel, confère au cône de croissance une réponse au signal répulsif.

réponse à Sema3B ainsi créée. Cela suggérait que le signal majeur de régulation n'est pas NrCAM [8].

\section{GDNF : un régulateur de la} signalisation de la sémaphorine $3 B$ Nous avons donc cherché à identifier ce signal, et nous nous sommes intéressés à GDNF, dont la restriction d'expression à la plaque du plancher dans la moelle épinière en développement est particulièrement frappante. Nous n'avons pas détecté sur les axones commissuraux les effets chimioattractif et promoteur de croissance typiques du GDNF. En revanche, nous avons observé, dans divers modèles de cultures neuronales, que le GDNF est capable d'inhiber l'activité de la calpaïne l, et donc de restaurer l'expression de PlexAl dans le cône de croissance des axones commissuraux et la réponse répulsive à Sema3B (Figure 2). De plus, nous avons observé que l'invalidation de GDNF chez la souris génère des défauts de traversée de la plaque du plancher qui ressemblent à ceux qui sont induits par la perte de la signalisation Sema3B/PlexAl. Ces défauts étaient toutefois atténués, ce qui suggérait l'action conjointe du GDNF et de NrCAM dans l'activation de la réponse répulsive à Sema3B. Pour valider cette hypothèse, nous avons généré une lignée de souris dont les gènes codant pour GDNF et NrCAM étaient invalidés : les défauts de traversée étaient cette fois aussi proéminents que ceux qu'entraîne la perte de Sema3B ou PlexAl [9]. Classiquement, le GDNF exerce ses effets via deux récepteurs de signalisation principaux : la tyrosine kinase RET (rearranged during transfection) et NCAM. RET et NCAM recrutent toutes deux un corécepteur, GFRal (GDNF family receptor $\alpha 1$ ), qui augmente l'affinité de liaison de GDNF et l'activation des voies de signalisation intracellulaires $[2,4]$. Nous avons observé, par immunomarquages sur coupes et analyse d'une lignée murine dans laquelle une étiquette fluorescente a été fusionnée à RET, que ce récepteur n'est pas présent dans les axones des neurones commissuraux. Au contraire, NCAM et GFR $\alpha$ l ont été détectés dans les axones aux stades de pré-traversée, traversée et posttraversée. L'analyse de la trajectoire des projections commissurales dans la lignée murine déficiente pour RहT n'a révélé aucun défaut de traversée de la plaque du plancher, au contraire de la lignée mutante pour NCAM, qui reproduit les défauts attendus d'une perte d'activité de GDNF. Nous avons également observé, dans les modèles de cultures neuronales, que la perte de NCAM et GFR $\alpha$, et non celle de RET, compromet la mise en place du mécanisme de gain de réponse à Sema3B, c'est-àdire l'inhibition de l'activité calpaïne, l'expression de surface de PlexAl et la réponse répulsive à Sema3B. Ces différentes approches expérimentales ont donc permis de conclure que le médiateur de GDNF dans ce système biologique est le complexe NCAM/GFRol (Figure 2). Ces résultats permettent d'enrichir la palette des fonctions du facteur GDNF et d'identifier de nouveaux intervenants dans les voies de contrôle de la signalisation sémaphorine et les modifications post-traductionnelles des récepteurs plexine. PlexAl est un récepteur partagé par de nombreuses sémaphorines, sécrétées (Sema3A à Sema3G) comme transmembranaires (Sema5). Outre leur rôle lors du développement du système nerveux, les signalisations sémaphorine/ plexine exercent de nombreuses autres fonctions : elles participent à la formation de nombreux organes et tissus comme le cœur, le système vasculaire et l'os [10]; chez l'adulte, elles interviennent dans les fonctions immunitaires [11]. L'altération de leur expression ou de leurs propriétés contribue à la dissémination des cellules cancéreuses, à la vascularisation des tumeurs, ou encore à la limitation de la régénération du système nerveux lésé [12]. Cette étude ouvre ainsi des perspectives thérapeutiques car l'ensemble de ce 
réseau de protéines est impliqué dans de multiples contextes physiopathologiques. De nouvelles questions se posent à présent : cette propriété de GDNF estelle partagée par d'autres facteurs neurotrophiques? la régulation de PlexAl intervient-elle également dans d'autres récepteurs plexines? Si tel était le cas, le spectre d'interactions fonctionnelles entre ces différentes grandes familles de protéines en serait élargi. $\diamond$

The neurotrophic factor GDNF, a novel modulator of the semaphorin signaling pathway during axon guidance

\section{LIENS D'INTÉRÊT}

Les auteurs déclarent n'avoir aucun lien d'intérêt concernant les données publiées dans cet article.

\section{RÉFÉRENCES}

1. Bermingham N, Hillermann R, Gilmour F, et al. Human glial cell line-derived neurotrophic factor (GDNF) maps to chromosome 5. Hum Genet 1995 ; $96: 671-3$.

2. Saavedra A, Baltazar G, Duarte EP. Driving GDNF expression: the green and the red traffic lights. Prog Neurobiol 2008 ; 86 : 186-215.

3. Rangasamy SB, Soderstrom K, Bakay RA, Kordower JH. Neurotrophic factor therapy for Parkinson's disease. Prog Brain Res 2010 ; $184: 237-64$.

4. Paratcha G, Ledda F. GDNF and GFRalpha: a versatile molecular complex for developing neurons. Trends Neurosci 2008 ; 31 : 384-91.

5. Charron F, Tessier-Lavigne M. Novel brain wiring functions for classical morphogens: a role as graded positional cues in axon guidance. Development 2005 ; $132: 2251-62$

6. Chédotal, A. Further tales of the midline. Curr Opin Neurobiol $2011 ; 2: 68-75$.

7. Evans TA, Bashaw GJ. Axon guidance at the midline: of mice and flies. Curr Opin Neurobiol $2010 ; 1: 79-85$.

\section{NOUVELL $\varepsilon$}

Un canal ionique qui protège
la peau de la pression

Bérengère Fromy ${ }^{1,2}$, Éric Lingueglia ${ }^{3,4,5}$,

Dominique Sigaudo-Roussel ${ }^{1,2}$, Jean-Louis Saumet ${ }^{1,2}$,

Michel Lazdunski $i^{3,4}$

$>$ En l'absence d'ajustements appropriés de la microcirculation cutanée, la peau est en danger face à une pression, même légère, avec un risque d'escarres important. Des processus biologiques particuliers permettent à une peau saine de résister aux contraintes mécaniques, en particulier grâce à la capacité de vasodilatation des microvaisseaux de la peau qui permet l'augmentation du flux sanguin cutané lorsqu'une pression faible lui est appliquée [1]. Cette vasodilatation est appelée PIV, pour pressure-induced vasodilation.

Les sujets touchés par le vieillissement ou atteints de diabète [2, 3] sont privés de cette PIV et présentent une baisse précoce du flux sanguin cutané dès l'application de très faibles pressions, reflétant une fragilité vasculaire qui peut augmenter le risque d'escarres ou de plaies ischémiques de pression.

Notre objectif était d'identifier un mécanorécepteur nerveux cutané qui déclenche la PIV. Les canaux ASIC (acid-sensing ion channels) [4] sont de bons candidats pour participer à la transduction mécanique dans la PIV, même si leur rôle comme mécanosenseur nerveux reste controversé $[5,6,12]$. Ces canaux appartiennent à la famille des canaux sodium sensibles à l'amiloride et des dégénérines (famille $\varepsilon N a C / D E G)$ qui comprend entre autres le canal sodium épithélial ( $\mathrm{ENaC})$. ASIC3, une des trois sous-unités, était un candidat particulièrement intéressant : (1) il est très exprimé dans les neurones sensoriels ; (2) il est présent dans les terminaisons nerveuses à des sites où les stimulus mécaniques sont convertis en signaux électriques ; de plus,
8. Nawabi H, Briançon-Marjollet A, Clark C, et al. A midline switch of receptor processing regulates commissural axon guidance in vertebrates. Genes Dev $2010 ; 15: 396-410$.

9. Charoy C, Nawabi H, Reynaud F, et al. gdnf activates midline repulsion by Semaphorin3B via NCAM during commissural axon guidance. Neuron $2012 ; 75$ : 1051.

10. Kruger RP, Aurandt J, Guan KL. Semaphorins command cells to move. Nat Rev Mol Cell Biol $2005 ; 6$ : 789-800.

11. Takamatsu H, Kumanogoh A. Diverse roles for semaphorin-plexin signaling in the immune system. Trends Immunol $2012 ; 33$ : 127-35.

12. Roth $L$, Koncina $\varepsilon$, Satkauskas $S$, et al. The many faces of semaphorins: from development to pathology. Cell Mol Life Sci 2009 ; 66 : 649-66.
${ }^{I}$ Institut de biologie et chimie des protéines, CNRS-FRE 3310, 7, passage du Vercors, 69367, Lyon Cedex 07, France.

${ }^{2}$ Université de Lyon 1, Lyon, France.

${ }^{3}$ Institut de pharmacologie moléculaire et cellulaire, CNRS, UMR 7275, Valbonne, France. ${ }^{4}$ Université de Nice-Sophia Antipolis, UMR 7275, Valbonne, France. ${ }^{5}$ LabEx ICST Valbonne, France. berengere.fromy@univ-lyonl.fr

la mécanosensibilité est altérée chez des souris déficientes en ASIC3 (knockout) [7] ; (3) il est coexprimé avec le CGRP (calcitonin gene-related peptide) - un peptide vasodilatateur indispensable à la PIV [8] - dans la catégorie des neurones sensoriels dont certains innervent les vaisseaux sanguins [9]. Pour toutes ces raisons, nous avons étudié le rôle d'ASIC3 dans la PIV et la survenue des escarres.

Le double rôle d'ASIC3 dans la protection contre les lésions induites par la pression cutanée

\section{ASIC3 détecte les variations de pression cutanée}

Afin de décrire précisément le rôle du canal ASIC3 dans la PIV, nous avons étu- 